\title{
ANALYSIS OF A DOMAIN DECOMPOSITION METHOD FOR THE NEARLY ELASTIC WAVE EQUATIONS BASED ON MIXED FINITE ELEMENT METHODS *
}

\author{
XiaObing FEng†
}

\begin{abstract}
This paper concerns the numerical solutions of the nearly elastic wave equations with the first order absorbing boundary condition; these equations describe the motion of a nearly elastic solid in the frequency domain. Two mixed finite elements, the Johnson-Mercier element and the Arnold-Douglas-Gupta element, are adapted and analyzed for the problem. The resulting mixed finite element equations are complex-valued and are neither Hermitian nor definite. As a result, most standard it erative methods fail to converge for the systems. To solve the mixed finite element equations, a parallelizable domain decomposition iterative method is proposed. The convergence of the method is demonstrated and a rate of convergence of the form $1-C h$ is derived. These results are valid for the case when the original domain is decomposed into subdomains which consist of an individual element associated with the above two mixed finite elements.
\end{abstract}

\$1. Introduction. Wave propagation in real media is affected by attenuation and dispersion. Therefore, a realistic simulation of wave propagation in the media should be able to reproduce these two effects. Attenuation and dispersion associated with wave transmission are often better described when problems are formulated in the space-frequency domain as opposed to the space-time domain (Hamilton (1972), Johnston, Toksöz \& Timur (1978), White (1965)). In particular, attenuation that is nonlinear in the frequency leads to a pseudo-differential formulation in the space-time domain in place of a differential formulation in the space-frequency domain. To model wave propagation through an infinite domain, it is well known that a suitable radiation condition at infinity must complement the governing wave equations. Such a wave problem can be solved numerically by first truncating the given unbounded domain, imposing a suitable outgoing radiation condition on the (artificial) boundaries of the truncated domain, and then solving the resulting problems using finite difference methods, finite element methods, or other discretization methods.

In this paper we are interested in obtaining numerical solutions for the nearly elastic wave equations which govern the motion of a nearly elastic solid. "Nearly elastic solid" refers to a class of elastic materials whose constitutive relations allow the inclusion of dissipative effects via the use of complex Lamé constants (White (1965) and §2). To avoid using pseudo-differential

1991 Mathematics Subject Classification. 65N30,65N55.

Key words and phrases. mixed finite element methods, domain decomposition methods, nearly elastic wave equations.

†Department of Mathematics, The University of Tennessee, Knoxville, TN 37996, USA, xfeng@math.utk.edu.

${ }^{*}$ to appear in IMA Journal of Numerical Analysis. 
formulations, the constitutive relations are often given in the frequency domain. As a result, we have to numerically solve a sequence of noncoercive and Helmholtz-like elliptic systems.

The objective of this paper is to analyze some mixed finite element methods for the nearly elastic wave equations complemented with the first order absorbing boundary condition, and to develop a domain decomposition iterative method which is efficient for solving the mixed finite element systems. Mixed finite elements are chosen to discretize the wave equations because they give better approximations for the stress tensor, which is of significant importance in applications. The use of a domain decomposition iterative method for solving the mixed finite element equations has two motivating factors. First, classical relaxation methods such as Jacobi and SOR methods are not convergent for the problem. Second, domain decomposition methods can be implemented naturally on a parallel computer by assigning each subdomain to its own processor.

Unlike the situation for scalar second order elliptic problems, the pool of mixed finite elements for elasticity is very small (Brezzi \& Fortin (1991)). In this paper two mixed finite elements, the Johnson-Mercier element (Johnson \& Mercier (1978)) and the Arnold-DouglasGupta element (Arnold, Douglas \& Gupta (1984)) are adapted with slight modifications to discretize the nearly elastic wave equations. The domain decomposition method to be introduced later in this paper belongs to the non-overlapping class. It is well known that the main issue for constructing a domain decomposition method is how to pass information between subdomains (see Bramble, Pasciak, Wang \& Xu (1991), Glowinski \& Wheeler (1988), Marini \& Quarteroni (1989), Lions (1990), Widlund (1992), Xu (1992) and the references therein). In this paper our approach is to use a Robin type boundary condition as the information transmission condition on the subdomain interfaces. The idea of employing Robin type boundary conditions as interface conditions was first used by P. L. Lions in Lions (1990) for coercive elliptic problems, and later it was generalized to the scalar Helmholtz problem by B. Després in Després (1991). In both Lions (1990) and Després (1991) the domain decomposition methods were analyzed only for the differential problems; it is not trivial to apply this idea to the corresponding discrete problems. For the coercive elliptic problems, two successful approaches were reported recently in Douglas, Paes Leme, Roberts \& Wang (1993) and Le Tallec \& Sassi (1995), respectively. Douglas, Paes Leme, Roberts \& Wang (1993) proposed a discrete version of the Robin transmission condition for mixed finite element methods by making strong use of hybridization of mixed finite element methods. Convergence and the rate of convergence for the domain decomposition method were established. Le Tallec \& Sassi (1995) presented the domain decomposition method based on an augmented Lagrangian formulation using an appropriate positive self-adjoint non-local interface operator from $H^{\frac{1}{2}}$ on the union of the interfaces to its dual space. An $h$-independent rate of convergence for the sequence of Lagrangian multipliers was shown. For the noncoercive Helmholtz and Helmholtz-like problems, all known convergence results in the literature were given for the differential problems (see Després (1991), Feng (1992) and references therein); these papers presented only numerical results to validate the domain decomposition procedures for the discrete case. For the nearly elastic wave equations discretized by the well-known nonconforming Wilson finite element, Bennethum and the author 
(Bennethum \& Feng (1997) ) recently proposed a discrete Robin type transmission condition and an associated domain decomposition method using an interface interpolation technique. Both convergence and a convergence rate of the form $1-C h$ were established. The purpose of this paper is to search for efficient discrete Robin type transmission conditions and to give a rigorous analysis of the associated domain decomposition method for the mixed finite element discretization of the nearly elastic wave equations. Our analysis is based on the combination of the ideas from Després (1991) and Douglas, Paes Leme, Roberts \& Wang (1993).

The layout of this paper is as follows. Section 2 states the problem and collects some preliminaries. Section 3 presents the domain decomposition iterative procedure for the differential problem which is based on the mixed weak formulation; convergence of the procedure is demonstrated. Section 4 introduces the domain decomposition procedure for mixed finite element discretizations of the problem using the Johnson-Mercier element (Johnson \& Mercier (1978) ) and the Arnold-Douglas-Gupta elements (Arnold, Douglas \& Gupta (1984)). The convergence of the discrete iterative procedure is demonstrated in the case when the domain is decomposed into subdomains in which each subdomain consists of an individual element associated with the mixed elements mentioned above. For the same decomposition, we show that the rate of convergence of the discrete procedure has an upper bound of the form $1-C h$ for both mixed finite element methods.

§. Statement of the problem and preliminaries. Consider the following sequence of elliptic systems

$$
\begin{aligned}
-\rho \omega^{2} u-\operatorname{div} \underset{\sim}{\sigma(u)}=\underset{\sim}{f}, & \text { in } \Omega, \\
\underset{\sim}{\sigma(u)} \underset{\sim}{\nu}+i \underset{\sim}{\operatorname{si}} u=\underset{\sim}{g}, & \text { on } \Gamma=\partial \Omega,
\end{aligned}
$$

for each $\omega>0$ where $\Omega$ is a convex polygonal domain in $\mathbb{R}^{N}$ for $N=2,3$. In particular, we are interested in the case that $\Omega=(0,1)^{N}$. $\nu$ denotes the outward normal vector on $\Gamma$. $\rho$ denotes the density of the solid which, without loss of generality, is set to be one for simplicity. $\sim_{\sim}^{u}$ is the displacement vector in the frequency domain. The stress-strain relation in the frequency domain is described as follows

$$
\begin{aligned}
& \underset{\approx}{\sigma}=\lambda \operatorname{tr}(\underset{\approx}{\varepsilon}(u)) \underset{\sim}{I}+2 \mu \underset{\approx}{\varepsilon}(u), \quad \text { in } \Omega, \\
& \underset{\sim}{\varepsilon}(u)=\frac{1}{2}\left(\nabla \underset{\sim}{u}+\nabla \underset{\sim}{u^{T}}\right), \quad \text { in } \Omega, \\
& \lambda=\lambda_{r}+i \lambda_{i}, \quad \mu=\mu_{r}+i \mu_{i},
\end{aligned}
$$

where $\underset{\approx}{I}$ denotes the $N \times N$ identity matrix. The coefficients $\lambda_{r}$ and $\mu_{r}$ are known as the Lamé constants for the material. We assume that $\lambda_{i}$ and $\mu_{i}$ are strictly positive, $\lambda_{i}<<\lambda_{r}$ and $\mu_{i}<<\mu_{r}$. The coefficients $\lambda_{i}$ and $\mu_{i}$ are not directly measurable but are related to other parameters measuring attenuation. For their precise definitions and estimates, see Ravazzoli, Douglas, Santos \& Sheen (1992) and White (1965). $f$ denotes the external source vector in the 
frequency domain and $\underset{\tilde{\sim}}{A}$ is a given $N \times N$ positive definite constant matrix which takes the following form for $N=\tilde{\tilde{2}}$

$$
A=\left(\begin{array}{cc}
\nu_{1} & \nu_{2} \\
\nu_{2} & -\nu_{1}
\end{array}\right)\left(\begin{array}{cc}
\alpha & 0 \\
0 & \beta
\end{array}\right)\left(\begin{array}{cc}
\nu_{1} & \nu_{2} \\
\nu_{2} & -\nu_{1}
\end{array}\right)
$$

and

$$
\alpha=\sqrt{\frac{\lambda_{r}+2 \mu_{r}}{\rho}}, \quad \beta=\sqrt{\frac{\mu_{r}}{\rho}} .
$$

Condition (2.1.ii) with $g=0$ is a standard first order absorbing boundary condition; waves striking the boundary $(\tilde{\Gamma})$ normally are completely annihilated (Feng (1992), Ravazzoli, Douglas, Santos \& Sheen (1992)).

When $\lambda_{i}$ and $\mu_{i}$ vanish, the solid becomes an elastic material and (2.1) is the Fouriertransformation (in time) of the following classical elastic wave propagation equations

$$
\begin{array}{ll}
\rho \underset{\sim}{\rho} u_{t t}-\operatorname{div} \underset{\sim}{\sigma(u)}=\underset{\sim}{f}, & \text { in } \Omega \times[0, \infty), \\
\underset{\sim}{A u_{t}}+\underset{\sim}{\sigma(u) \underset{\sim}{\sim} \underset{\sim}{\sim}=g,} & \text { on } \Gamma \times[0, \infty), \\
\underset{\sim}{u}=u_{\sim}=0, & \text { in } \Omega \times\{0\} .
\end{array}
$$

Hence the frequency domain formulations for elastic waves are also included in (2.1) and they can be regarded as limiting forms of nearly elastic waves as $\lambda_{i}$ and $\mu_{i}$ go to zero.

The standard space notations will be used in the rest of this paper. For example, $H^{k}(\Omega)$ and $\|\cdot\|_{k, \Omega}(k=-1,0,1,2)$ denote the usual complex Sobolev space and its norm; $H^{s}(\Gamma)$ ( $s=0, \pm \frac{1}{2}$ ) and $\|\cdot\|_{s, \Gamma}$ denote the usual Sobolev space and its norm on the boundary $\Gamma$ of $\Omega$. For more details of the description these Sobolev spaces, see Brezzi \& Fortin (1991) and Ciarlet (1978).

Given any space $X, \underset{\sim}{X}$ [respectively, $\underset{\approx}{X}]$ denotes the space of $N$-vectors $[N \times N$-tensors] with components in $X$. If $X$ is normed, associated norms are defined by

$$
\|\underset{\sim}{v}\|_{\sim}=\left(\sum_{j=1}^{N}\left\|v_{j}\right\|_{X}^{2}\right)^{\frac{1}{2}}, \quad\|\underset{\sim}{\underset{\sim}{\tau}}\|_{\sim}=\left(\sum_{j, k=1}^{N}\left\|\tau_{j k}\right\|_{X}^{2}\right)^{\frac{1}{2}},
$$

and the subspace $\underset{\approx}{X}$ of $\underset{\approx}{X}$ consists of $N \times N$-tensors which are symmetric. Finally, we introduce the following special space notations

$$
V=L^{2}(\Omega), \quad \underset{\approx}{H}=\underset{\approx}{H}(\operatorname{div} ; \Omega)_{s}=\left\{\underset{\approx}{\tau} \in \underset{\approx}{L^{2}}(\Omega)_{s}: \operatorname{div} \underset{\approx}{\tau} \in \underset{\sim}{V}\right\} .
$$

The norm of $\underset{\approx}{H}$ is given by

$$
\|\underset{\approx}{\|}\|_{\underset{\sim}{H}}=\sqrt{\|\underset{\sim}{\|}\|_{V}^{2}+\|\operatorname{div} \underset{\sim}{\tau}\|_{V}^{2}}
$$


For each $\omega>0$, define the sesquilinear form $\Lambda_{\omega}(\cdot, \cdot): \underset{\sim}{H^{1}}(\Omega) \times \underset{\sim}{H^{1}}(\Omega) \rightarrow \mathbb{C}$,

$$
\begin{aligned}
& \left.\Lambda_{\omega}(\underset{\sim}{u}, \underset{\sim}{v})=(\underset{\approx}{\sigma} \underset{\sim}{u}), \underset{\approx}{\varepsilon}(v)\right)_{\Omega}-\omega^{2}(\underset{\sim}{u}, \underset{\sim}{v})_{\Omega}+i \omega\langle\underset{\approx}{A} u, \underset{\sim}{v}\rangle_{\Gamma} \\
& =\left(\lambda_{r} \nabla \cdot \underset{\sim}{u}, \nabla \cdot \underset{\sim}{v}\right)_{\Omega}+2\left(\mu_{r} \underset{\approx}{\varepsilon}(u), \underset{\sim}{\varepsilon}(v)\right)_{\Omega}-\omega^{2}(\underset{\sim}{w}, \underset{\sim}{v})_{\Omega} \\
& +i\left[\left(\lambda_{i} \nabla \cdot \underset{\sim}{u}, \nabla \cdot \underset{\sim}{v}\right)_{\Omega}+2\left(\mu_{i} \underset{\approx}{\varepsilon}(u), \underset{\sim}{\varepsilon}(\underset{\sim}{v})\right)_{\Omega}+\omega\langle\underset{\sim}{A w} \underset{\sim}{v} \underset{\sim}{v}\rangle_{\Gamma}\right],
\end{aligned}
$$

where $(\cdot, \cdot)_{\Omega}$ denotes the complex $L^{2}(\Omega)$ product and $\langle\cdot, \cdot\rangle_{\Gamma}$ denotes the duality between $\underset{\sim}{H}{ }^{\frac{1}{2}}(\Gamma)$ and $\underset{\sim}{H^{-\frac{1}{2}}}(\Gamma) ;(\cdot, \cdot)_{\Gamma}$ and $\left\langle\cdot, \cdot>_{\Omega}\right.$ denote the complex $L^{2}(\Gamma)$ product and duality between $\underset{\sim}{H^{1}}(\Omega)$ and $\underset{\sim}{H}{ }^{-1}(\Omega)$.

Definition 2.1. A vector-valued function $\underset{\sim}{u} \in \underset{\sim}{H^{1}}(\Omega)$ is said to be a weak solution of (2.1) if it satisfies the following equation

$$
\left.\Lambda_{\omega}(\underset{\sim}{u, v})=<\underset{\sim}{f}, \underset{\sim}{v}>_{\Omega}+\underset{\sim}{g}, \underset{\sim}{v}\right\rangle_{\Gamma}, \quad \forall \underset{\sim}{v} \in \underset{\sim}{H^{1}}(\Omega)
$$

Theorem 2.1. For any given $\underset{\sim}{f} \in \underset{\sim}{H^{-1}}(\Omega)$ and $\underset{\sim}{g} \in \underset{\sim}{H^{-\frac{1}{2}}}(\Gamma)$, problem (2.1) admits a unique solution $\underset{\sim}{u} \in \underset{\sim}{H^{-1}}(\Omega)$. Moreover, if $\underset{\sim}{f} \in \underset{\sim}{L^{2}}(\Omega)$ and $\underset{\sim}{g} \in \underset{\sim}{L^{2}}(\Gamma)$, then $\underset{\sim}{u} \in \underset{\sim}{H}{ }^{\frac{3}{2}}(\Omega)$.

Remark. A detailed proof of Theorem 2.1 is given in Bennethum \& Feng (1997). Application of the Fredholm Alternative Principle yields unique solvability in $\underset{\sim}{H^{-1}}(\Omega)$; the Gårding's inequality follows directly from the well-known Korn's inequality (see Lemma 2.1 below). The second part of the theorem follows from a recent result of Dahlberg, Kenig \& Verchota (1988) for the systems of elastostatics in Lipschitz domains.

Lemma 2.1. There exists a constant $C>0$ which depends only on $\Omega$ such that

$$
\|\underset{\sim}{u}\|_{1, \Omega}^{2} \leq C\left[\|\underset{\sim}{\varepsilon}(\underset{\sim}{u})\|_{0, \Omega}^{2}+\|\underset{\sim}{u}\|_{0, \Omega}^{2}\right]
$$

for any $\underset{\sim}{u} \in \underset{\sim}{H^{1}}(\Omega)$.

For a proof of Lemma 2.1, we refer to Duvaut \& Lions (1976).

Next we will derive an equivalent mixed weak formulation for (2.1). This will be done by introducing the stress tensor $\underset{\approx}{\sigma}$ as an additional independent unknown variable so that $\underset{\sim}{u}$ and $\underset{\approx}{\sigma}$ will be found simultaneously by solving the mixed formulation of the problem. For more on the theory of mixed methods, we refer to Brezzi \& Fortin (1991) and Ciarlet (1978).

Applying the matrix trace operator tr to both sides of $(2.1 . i)$ and solving for $\operatorname{the} \operatorname{tr}(\underset{\sim}{\varepsilon}(u))$, we see that

$$
\operatorname{tr}(\underset{\sim}{\varepsilon}(\underset{\sim}{u}))=\gamma \operatorname{tr}(\underset{\sim}{u}), \quad \text { where } \gamma= \begin{cases}\frac{\lambda}{4 \mu(\lambda+\mu)}, & \text { if } N=2, \\ \frac{\lambda}{2 \mu(3 \lambda+2 \mu)}, & \text { if } N=3 .\end{cases}
$$


Substituting (2.6) into (2.2) we get

$$
\frac{1}{2 \mu} \sigma \underset{\approx}{\sigma}-\gamma \operatorname{tr}(\underset{\approx}{\sigma}) \underset{\approx}{I}-\underset{\approx}{\varepsilon}(u)=0, \quad \text { in } \Omega
$$

Problem (2.1) can therefore be equivalently formulated as

$$
\begin{aligned}
& \frac{1}{2 \mu} \sigma-\gamma \operatorname{tr}(\underset{\approx}{\sigma}) \underset{\approx}{I}-\underset{\approx}{\varepsilon}(u)=0, \quad \text { in } \Omega, \\
& \operatorname{div} \underset{\approx}{\sigma}+\omega^{2} u=f, \quad \text { in } \Omega, \\
& \underset{\sim \sim}{\sigma \nu}+i \omega \underset{\sim}{A} u=\underset{\sim}{\sim}, \quad \text { on } \Gamma \text {. }
\end{aligned}
$$

The mixed weak formulation of $(2.1)$ is defined by seeking $\underset{\sim}{\sigma}, \underset{\sim}{u}) \in \underset{\approx}{H} \times \underset{\sim}{V}$ such that

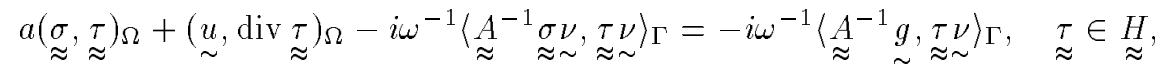

$$
\begin{aligned}
& (\operatorname{div} \underset{\approx}{\sigma}, \underset{\sim}{v})_{\Omega}+\omega^{2}(\underset{\sim}{u}, \underset{\sim}{v})_{\Omega}=-(\underset{\sim}{f}, \underset{\sim}{v})_{\Omega}, \quad \underset{\sim}{v} \in \underset{\sim}{V}
\end{aligned}
$$

where

$$
a(\underset{\approx}{\sigma}, \underset{\approx}{\tau})_{\Omega}=\int_{\Omega}\left[\frac{1}{2 \mu} \underset{\approx}{\sigma} \bar{\tau}-\gamma \operatorname{tr}(\underset{\approx}{\sigma}) \operatorname{tr}(\underset{\approx}{\tau})\right] d x
$$

The following theorem establishes unique solvability of (2.4)

Theorem 2.2. Let $\underset{\sim}{u} \in \underset{\sim}{H}(\Omega)$ be the solution of (2.1). Then $\underset{\sim}{\sigma}(\underset{\sim}{\sigma}), \underset{\sim}{u}) \in \underset{\approx}{H} \times \underset{\sim}{V}$ is the unique solution of (2.8). Conversely, if $\underset{\approx}{\sigma}, u) \in \underset{\sim}{H} \times \underset{\sim}{V}$ is a solution of $(2.8)$, then $\underset{\sim}{u} \in \underset{\sim}{H_{\sim}^{1}}(\Omega)$ and it is the unique weak solution of (2.1).

Proof. Suppose that $\underset{\sim}{u} \in \underset{\sim}{H}{ }^{1}(\Omega)$ is the unique weak solution of $(2.1)$, then

$$
\left.(\underset{\sim}{\sigma} \underset{\sim}{u}), \underset{\approx}{\varepsilon}(v))_{\Omega}-\omega^{2}(\underset{\sim}{u} \underset{\sim}{v})_{\Omega}+i \omega \underset{\approx \sim}{A} \underset{\sim}{u} \underset{\sim}{v}\right\rangle_{\Gamma}=(\underset{\sim}{f} \underset{\sim}{v})_{\Omega}+\langle\underset{\sim}{g} \underset{\sim}{v}\rangle_{\Gamma}, \underset{\sim}{\forall v} \in \underset{\sim}{H^{1}}(\Omega) .
$$

Clearly, $\left.\underset{\sim}{u}\right|_{\Gamma} \in \underset{\sim}{H}{ }_{\sim}^{\frac{1}{2}}(\Gamma)$. Let $\underset{\approx}{\sigma}=\underset{\approx}{\sigma} \underset{\sim}{u}$, which is defined by $(2.2)$. To show $\underset{\approx}{\sigma} \in \underset{\approx}{H} \equiv \underset{\sim}{H}(\operatorname{div}, \Omega)_{s}$, it suffices to show that $\operatorname{div} \underset{\approx}{\sigma} \in \underset{\sim}{L^{2}}(\Omega)$.

First, notice that $\operatorname{div} \underset{\approx}{\sigma}$ is a distribution, hence for any $\varphi \in \underset{\sim}{\mathcal{D}}=C_{0}^{\infty}(\Omega)$,

$$
\begin{array}{rlr}
<\operatorname{div} \underset{\approx}{\sigma}, \varphi>{ }_{\sim}> & =-<\underset{\approx}{\sigma} \underset{\approx}{\varepsilon}(\varphi)>=-(\underset{\sim}{\sigma}, \underset{\sim}{\varepsilon}(\underset{\sim}{\varphi}))_{\Omega} & \\
& =-\omega^{2}(\underset{\sim}{u, \varphi})_{\Omega}-(\underset{\sim}{f}, \underset{\sim}{\varphi})_{\Omega} & \text { by }(2.9) .
\end{array}
$$

There exists a positive constant $C \underset{\sim}{u} \underset{\sim}{f})$ such that

$$
|<\operatorname{div} \underset{\approx}{\sigma}, \underset{\sim}{\varphi}>| \leq C \underset{\sim}{u}, \underset{\sim}{f})\|\underset{\sim}{\varphi}\|_{0, \Omega}
$$

(2.11) and density of $\underset{\sim}{\mathcal{D}}$ in $\underset{\sim}{L^{2}}(\Omega)$ implies that $\operatorname{div} \underset{\approx}{\sigma} \in \underset{\sim}{L^{2}}(\Omega)$ and $\underset{\approx}{\sigma} \in \underset{\sim}{H}(\operatorname{div}, \Omega)_{s} \equiv \underset{\approx}{H}$. 
Next, we will show that $(\underset{\sim}{\sigma}, u)$ also satisfies the equations (2.8.i) and (2.8.ii). (2.8.ii) immediately follows from (2.10). By the definition of $\underset{\approx}{\sigma},(2.7 . i)$ holds for $\underset{\sim}{\sigma}$, so (2.8.i) follows from applying an integration by parts to (2.7.i), (2.9) and the following identity

$$
\begin{aligned}
& (\operatorname{div} \underset{\sim}{\sigma}, \underset{\sim}{v})_{\Omega}=-(\underset{\sim}{\sigma}, \underset{\sim}{\varepsilon}(v))_{\Omega}+\langle\underset{\sim \sim}{\sigma \nu}, \underset{\sim}{v}\rangle_{\Gamma} \\
& \left.=\left(-\omega^{2} u+\underset{\sim}{f}, \underset{\sim}{v}\right)_{\Omega}+\underset{\sim \sim}{\langle\sigma} \underset{\sim}{\sim}+i \underset{\sim}{A} u-\underset{\sim}{g}, \underset{\sim}{v}\right\rangle_{\Gamma} .
\end{aligned}
$$

Conversely, let $\underset{\sim}{\sigma}, \underset{\sim}{u}) \in \underset{\approx}{H} \times \underset{\sim}{V}$ be a solution of $(2.8)$. We want to show that $\underset{\sim}{u} \in \underset{\sim}{H}{ }^{1}(\Omega)$ and that it satisfies $(2.9)$. For the distribution $\nabla \underset{\sim}{\sim}$, we have (by $(2.8 . i)$ )

$$
\begin{aligned}
<\underset{\sim}{\varepsilon(u)}(\underset{\sim}{u}), & =<\nabla \underset{\sim}{\nabla u}, \underset{\approx}{\tau}>=-<\underset{\sim}{u}, \operatorname{div} \underset{\approx}{\tau}> \\
& =-(\underset{\sim}{u}, \operatorname{div} \underset{\approx}{\tau})_{\Omega}=a(\underset{\approx}{\sigma}, \underset{\approx}{\tau})_{\Omega}, \quad \underset{\approx}{\forall} \in \underset{\approx}{\mathcal{D}} s .
\end{aligned}
$$

Hence, there exists a positive constant $C \underset{\approx}{\sigma})$ such that

$$
|<\underset{\sim}{\varepsilon}(u), \underset{\sim}{\tau}>| \leq C(\underset{\approx}{\sigma})\left\|\left.\right|_{\approx} ^{\tau}\right\|_{0, \Omega}, \quad \underset{\approx}{\forall} \in \underset{\sim}{\mathcal{D}} s
$$

Since $\underset{\sim}{\varepsilon}(u)$ is symmetric, the above inequality implies that $\underset{\sim}{\varepsilon}(u) \in \underset{\sim}{L^{2}}(\Omega)$. By Korn's inequality we conclude that $\underset{\sim}{u} \in \underset{\sim}{H}(\Omega)$. For $\underset{\sim}{u} \in \underset{\sim}{H}{ }^{1}(\Omega)$, we have (from $(2.13)$ )

$$
\left(\frac{1}{2 \mu} \sigma \underset{\approx}{\sigma}-\gamma \operatorname{tr}(\underset{\approx}{\sigma}) \underset{\approx}{I}, \underset{\approx}{\tau}\right)_{\Omega}=(\underset{\approx}{\varepsilon}(u), \underset{\approx}{\tau})_{\Omega}, \quad \underset{\approx}{\sim} \in \underset{\approx}{\mathcal{D}} s
$$

Noticing that $\underset{\approx}{\sigma} \underset{\approx}{\varepsilon}(u)$ and $\underset{\sim}{I}$ are symmetric, we see from (2.14) that the identity (2.7.i) holds for $\underset{\approx}{\sigma}$ and $\underset{\sim}{u}$. Hence, $\underset{\sim}{\sigma}=\underset{\approx}{\sigma(u)} \underset{\sim}{\sim}$. Substituting the identity

$$
(\underset{\sim}{u}, \operatorname{div} \underset{\approx}{\tau})_{\Omega}=\langle\underset{\sim}{u} \underset{\sim}{\tau} \cdot \underset{\sim}{\nu}\rangle_{\Gamma}-(\nabla \underset{\sim}{u}, \underset{\approx}{\tau})_{\Omega} \quad \underset{\approx}{\forall} \in \underset{\approx}{H}
$$

into (2.8.i) and using (2.7.i) we also get

$$
\underset{\sim}{u}=i \omega^{-1} A^{-1}(\underset{\sim}{\sigma \nu}-\underset{\sim}{g})
$$

Finally, choosing arbitrary $\underset{\sim}{v} \in \underset{\sim}{H^{1}}(\Omega)$ as a test function in (2.8.ii), applying an integration by parts to the first term, and using equation (2.15) we obtain

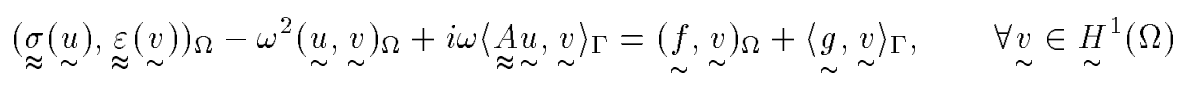

which is $(2.9)$. This completes the proof.

$\S 3$. The DD method for the differential problem. In this section we will introduce a non-overlapping domain decomposition iterative method for solving problem (2.1) based on the mixed weak formulation (2.8). The utility of this algorithm will be demonstrated by proving its convergence. For convenience and for physical considerations, we will henceforth assume that $\underset{\sim}{g}=0$. 
§3.1. The DD iterative algorithm. Let $\left\{\Omega_{j}\right\}_{j=1}^{J}$ be a non-overlapping partition of $\Omega$ with Lipschitz boundaries $\left\{\partial \Omega_{j}\right\}$. Introduce the notations

$$
\Gamma_{k}=\Gamma_{k 0}=\Gamma \cap \partial \Omega_{k}, \quad \text { and } \quad \Gamma_{k j}=\partial \Omega_{k} \cap \partial \Omega_{j} .
$$

Let $\underset{\sim}{u}$ denote the restriction of the solution $\underset{\sim}{u}$ on $\Omega_{j}$. It is well-known that $\underset{\sim}{u_{j}}$ must satisfy

$$
\begin{aligned}
& \frac{1}{2 \mu} \sigma_{\approx}-\gamma \operatorname{tr}\left(\underset{\approx}{\sigma_{k}}\right) \underset{\approx}{I}-\underset{\approx}{\varepsilon}\left(u_{k}\right)=0, \quad \text { in } \Omega_{k}, \\
& \operatorname{div} \underset{\approx}{\sigma_{k}}+\omega^{2} u_{\sim}=f, \quad \text { in } \Omega_{k}, \\
& \underset{\approx}{\sigma} \underset{\sim}{\nu_{k}}+i \omega \underset{\sim}{A u_{k}}=0, \quad \text { on } \Gamma_{k} \text {, }
\end{aligned}
$$

and the consistency conditions

$$
\begin{array}{ll}
u_{k}=\underset{\sim}{u_{j}}, & \text { on } \Gamma_{k j}, \\
\underset{\sim}{\sigma_{k}} \underset{\sim}{\nu_{k}}=-\underset{\approx}{\sigma_{j}}{ }_{\sim}^{\nu_{j}}, & \text { on } \Gamma_{k j} .
\end{array}
$$

Notice that the above two consistency conditions can be replaced by the following Robin type boundary conditions (Bennethum \& Feng (1997), Després (1991), Douglas, Paes Leme, Roberts \& Wang (1993), Lions (1990))

$$
\begin{aligned}
& \underset{\sim}{\sigma}{ }_{\sim}^{\nu_{k}}+\alpha \underset{\sim}{u_{k}}=-\underset{\sim}{\sigma}{\underset{\sim}{j}}_{j}+\alpha \underset{\sim}{u_{j}}, \quad \text { on } \Gamma_{k j}, \\
& \underset{\sim}{\sigma}{ }_{\sim} \nu_{j}+\alpha \underset{\sim}{u_{j}}=-\underset{\sim}{\sigma}{ }_{\sim} \nu_{k}+\alpha \underset{\sim}{u_{k}}, \quad \text { on } \Gamma_{k j},
\end{aligned}
$$

where $\alpha$ is a nonzero complex piecewise constant function on $\cup \Gamma_{k j}$. In this paper we choose $\alpha=-\alpha_{r}+i \alpha_{i}$ with $\alpha_{r}>0$ and $\alpha_{i}>0$. The reason for the restriction will be clear in the next subsection. It is easy to check that the problem (3.1) is equivalent to the following

$$
\begin{aligned}
& \frac{1}{2 \mu} \underset{\approx}{\sigma_{k}}-\gamma \operatorname{tr}\left(\underset{\approx}{\sigma_{k}}\right) \underset{\approx}{I}-\underset{\approx}{\varepsilon}\left(u_{k}\right)=0, \quad \text { in } \Omega_{k}, \\
& \operatorname{div} \underset{\sim}{\sigma} \sigma_{k}+\omega^{2}{\underset{\sim}{u}}_{k}=\underset{\sim}{f}, \quad \text { in } \Omega_{k}, \\
& \underset{\sim}{\sigma}{ }_{\sim} \nu_{k}+i \omega \underset{\sim}{A u_{k}}=0, \quad \text { on } \Gamma_{k}, \\
& \underset{\sim}{\sigma}{ }_{\sim} \nu_{k}+\alpha \underset{\sim}{u_{k}}=-\underset{\sim}{\sigma}{\underset{\sim}{j}}_{j}+\alpha \underset{\sim}{u_{j}}, \quad \text { on } \Gamma_{k j} \text {. }
\end{aligned}
$$

Let $\underset{\approx}{H_{k}}=\left.\underset{\approx}{H}\right|_{\Omega_{k}}$ and $\underset{\sim}{V_{k}}=\left.\underset{\sim}{V}\right|_{\Omega_{k}}$ Then the mixed weak formulation (2.1) over the partition $\left\{\Omega_{k}\right\}$ is found by seeking $\left.\underset{\sim}{\sim} \underset{\sim}{\sigma}, \underset{\sim}{\sigma_{u}}\right) \in \underset{\approx}{H_{k}} \times \underset{\sim}{V_{k}}, k, j=1, \ldots, J$, such that

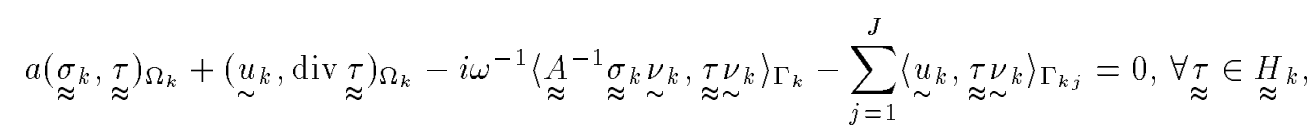

(3.4.ii) $\left(\operatorname{div} \underset{\sim}{\sigma} \sigma_{\sim}, \underset{\sim}{v}\right)_{\Omega_{k}}+\omega^{2}\left(\underset{\sim}{u_{k}}, \underset{\sim}{v}\right)_{\Omega_{k}}=-(\underset{\sim}{f}, \underset{\sim}{v})_{\Omega_{k}}$, $\forall \underset{\sim}{v} \in \underset{\sim}{V_{k}}$,

(3.4.iii) $\underset{\sim}{\sigma}{ }_{\sim}^{\nu_{k}}+\alpha \underset{\sim}{u_{k}}=-\underset{\sim}{\sigma}{ }_{\sim} \nu_{j}+\alpha \underset{\sim}{u_{j}}$, on $\Gamma_{k j}$. 
Lemma 3.1. Problem (2.8) is equivalent to problem (3.4) in the sense that $\left.\underset{\approx}{\sigma}\right|_{\Omega_{k}}=\underset{\approx}{\sigma_{k}}$ and

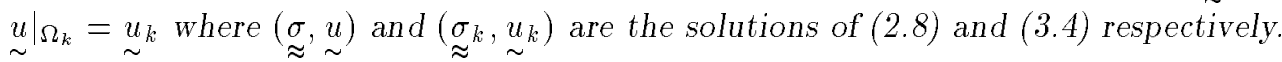

The objective of a domain decomposition iterative procedure is to localize the computation to problems over subdomains $\left\{\Omega_{k}\right\}$ of $\Omega$. There are many ways to do the localization (see Bramble, Pasciak, Wang \& Xu (1991), Després (1991), Douglas, Paes Leme, Roberts \& Wang (1993), Glowinski \& Wheeler (1988), Lions (1990), Widlund (1992), Xu (1992) and reference therein). In this section we localize the problem to each $\Omega_{k}$ by evaluating the quantities in (3.4) related to $\Omega_{k}$ at the new iterate level and those in (3.4) related to neighboring subdomains $\Omega_{j}$ such that $\Gamma_{k j} \neq \phi$ at the previous level. Specifically, the algorithm reads as follows

Choose $\left(\underset{\sim}{\sigma}, u_{\sim}^{0}\right) \in \underset{\sim}{\sigma_{k}} \times \underset{\sim}{V_{k}}$ with $\underset{\sim}{\sigma_{k}^{0}} \nu_{\sim} \in \underset{\sim}{L^{2}}\left(\Gamma_{k j}\right), k, j=1, \cdots J$, arbitrarily, then compute $\left(\underset{\approx}{\sigma}, u_{\sim}^{n}\right) \in \underset{\approx}{\underset{\sim}{H}} k \times \underset{\sim}{V} k$ for $n \geq 1$ recursively by solving

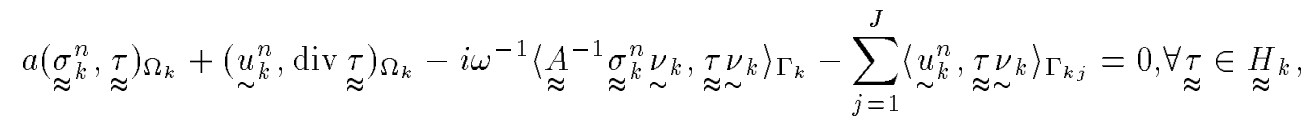

$$
\begin{aligned}
& \text { (3.5.ii) }(\operatorname{div} \underset{\sim}{\underset{\sim}{\sigma}}, \underset{\sim}{v})_{\Omega_{k}}+\omega^{2}\left(\underset{\sim}{u_{k}^{n}}, \underset{\sim}{v}\right)_{\Omega_{k}}=-(\underset{\sim}{f}, \underset{\sim}{v})_{\Omega_{k}} \text {, } \\
& \underset{\sim}{v} \in \underset{\sim}{V_{k}}
\end{aligned}
$$

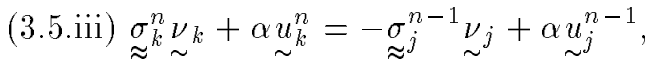

$$
\begin{aligned}
& \text { on } \Gamma_{k j} \text {. }
\end{aligned}
$$

Theorems 2.1 and 2.2 lead to the following lemma.

Lemma 3.2. (3.5) is uniquely solvable for each $n \geq 1$; the iterate sequence $\left\{\left({\underset{\sim}{\sigma}}_{k}^{n}, \underset{\sim}{u}\right)\right\}$ is well-defined.

§3.2. Convergence analysis. In this subsection, we will establish the utility of this domain decomposition iterative algorithm proposed earlier by proving the following convergence theorem.

Theorem 3.1. The solution $\left(\underset{\sim}{\sigma_{k}^{n}}, \underset{\sim}{u}\right)$ of (3.5) converges to the solution $\left.\underset{\sim}{\sigma}, \underset{\sim}{\sim}\right)$ of $(2.8)$ strongly in $\underset{\approx}{H_{k}} \times \underset{\sim}{V_{k}}$ provided that $\alpha_{r}>0, \alpha_{i}>0$ and $\alpha_{i} \mu_{i}>4 \alpha_{r} \mu_{r}$ in the parameter $\alpha=-\alpha_{r}+i \alpha_{i}$.

To show above theorem we need some auxiliary lemmas. Define the error functions

$$
\underset{\sim}{e_{k}^{n}}=\underset{\sim}{u_{k}^{n}}-\underset{\sim}{u_{k}}, \quad \underset{\approx}{\pi_{k}^{n}}=\underset{\approx}{\sigma} \sigma^{n}-\underset{\sigma_{k}}{\sigma}
$$

Due to the linearity of the equations, it follows from (3.4) and (3.5) that the error functions satisfy the following equations

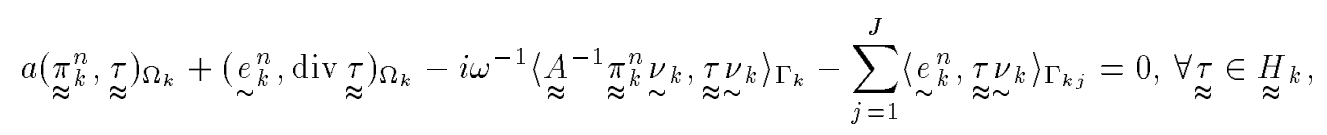

(3.7.ii) $\left(\operatorname{div} \underset{\approx}{\pi_{k}^{n}}, \underset{\sim}{v}\right)_{\Omega_{k}}+\omega^{2}(\underset{\sim}{e}, \underset{\sim}{n})_{\Omega_{k}}=0$ $\underset{\sim}{v} \in \underset{\sim}{V} k$ (3.7.iii) $\underset{\sim}{\pi_{k}^{n}}{\underset{\sim}{k}}_{k}+\alpha \underset{\sim}{e_{k}^{n}}=-\underset{\sim}{\pi_{j}^{n-1}} \nu_{\sim}+\alpha{\underset{\sim}{j}}_{j}^{n-1}$, on $\Gamma_{k j}$. 
Choosing $\underset{\sim}{\tau}={\underset{\sim}{k}}_{k}^{n}$ in (3.7.i) and $\underset{\sim}{v}={\underset{\sim}{j}}_{j}^{n}$ in (3.7.ii) and subtracting the resulting two equations yields

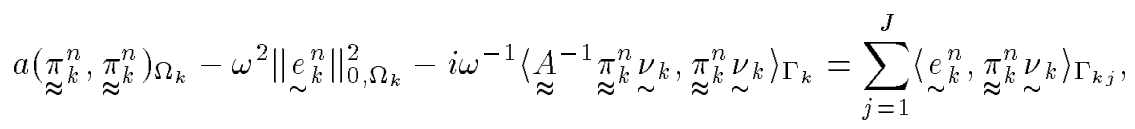

from which we immediately have

Lemma 3.3. The error functions $\underset{\sim}{e_{k}^{n}}$ and $\underset{\sim}{\pi}{ }^{n}$ satisfy the following identities

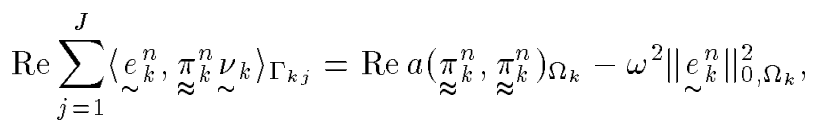

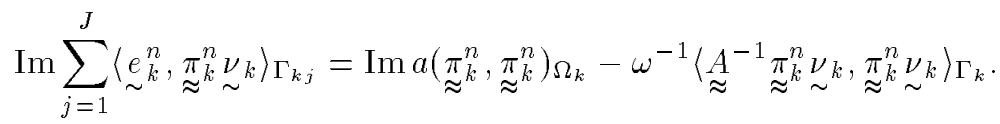

Define the "pseudo energy" $E(\underset{\approx}{\underset{\sim}{\pi}, e\}})$ by

$$
E(\{\underset{\sim}{\pi}, \underset{\sim}{e}\})=\sum_{k=1}^{J} \sum_{j=1}^{J} \int_{\Gamma_{k j}}\left|\underset{\sim}{\pi_{k}} \nu_{\sim}+\alpha \underset{\sim}{e_{k}}\right|^{2} d s
$$

and let $E^{n}=E\left(\left\{\underset{\sim}{\pi_{j}^{n}}, \underset{\sim}{e_{j}^{n}}, \underset{\sim}{\xi_{j k}^{n}}\right\}\right)$. Then we have

Lemma 3.4. The following equality holds

$$
\begin{aligned}
& E^{n+1}=E^{n}-4 \sum_{k=1}^{J}\left\{\alpha_{i}\left[\omega^{-1}\left\langle\underset{\approx}{A} \underset{\approx}{\pi_{k}^{n}}{\underset{\sim}{k}}_{k}, \underset{\approx}{\pi^{n}} \sim_{\sim}^{\nu_{k}}\right\rangle_{\Gamma_{k}}-\operatorname{Im} a\left(\underset{\approx}{\pi^{n}}, \underset{\approx}{\pi^{n}}\right)_{\Omega_{k}}\right]\right. \\
& \left.+\alpha_{r}\left[\omega^{2}\left\|{\underset{\sim}{k}}_{k}^{n}\right\|_{0, \Omega_{k}}^{2}-\operatorname{Re} a\left(\underset{\approx}{\pi_{k}^{n}}, \underset{\approx}{\pi_{k}^{n}}\right)_{\Omega_{k}}\right]\right\} .
\end{aligned}
$$

Proof. By (3.7.iii) and (3.8.i) we get

$$
\begin{aligned}
& E^{n+1}=\sum_{k=1}^{J} \sum_{j=1}^{J} \int_{\Gamma_{k j}}\left|{\underset{\approx}{k}}^{n+1}{\underset{\sim}{\nu}}_{k}+\alpha \underset{\sim}{e_{k}^{n+1}}\right|^{2} d s \\
& =\sum_{j=1}^{J} \sum_{k=1}^{J} \int_{\Gamma_{j k}}\left|-\underset{\sim}{\pi_{j}^{n}} \nu_{\sim}+\alpha \underset{\sim}{e_{j}^{n}}\right|^{2} d s \\
& =\sum_{j=1}^{J} \sum_{k=1}^{J} \int_{\Gamma_{j k}}\left[\left|{\underset{\sim}{j}}_{j}^{n} \nu_{\sim}\right|^{2}+|\alpha|^{2}\left|e_{\sim}^{n}\right|^{2}-2 \operatorname{Re}\left(\alpha \underset{\sim}{e_{j}^{n}} \overline{\pi^{n}} \overline{\nu_{j}}\right)\right] d s \\
& =E^{n}-4 \sum_{j=1}^{J} \operatorname{Re}\left[\alpha \sum_{k=1}^{J}\left\langle\underset{\sim}{e_{j}^{n}}, \underset{\sim}{\pi_{j}^{n}}{\underset{\sim}{j}}_{j}\right\rangle_{\Gamma_{j k}}\right] \\
& =E^{n}-4 \sum_{j=1}^{J}\left[-\alpha_{r} \operatorname{Re} \sum_{k=1}^{J}\left\langle\underset{\sim}{e_{j}^{n}}, \underset{\sim}{\pi_{j}^{n}} \nu_{\sim}\right\rangle_{\Gamma_{j k}}-\alpha_{i} \operatorname{Im} \sum_{k=1}^{J}\left\langle{\underset{\sim}{j}}_{j}^{n}, \underset{\sim}{\pi_{j}^{n}} \nu_{\sim}\right\rangle_{\Gamma_{j k}}\right] \\
& =E^{n}-4 \sum_{k=1}^{J}\left\{\alpha_{i}\left[\omega^{-1} \underset{\approx}{A^{-1}} \underset{\approx}{\pi_{k}^{n}} \nu_{\sim}, \underset{\approx}{\nu_{k}^{n}} \nu_{\sim}\right\rangle_{\Gamma_{k}}-\operatorname{Im} a\left(\underset{\approx}{\pi_{k}^{n}}, \underset{\approx}{\pi_{k}^{n}}\right) \Omega_{k}\right]
\end{aligned}
$$

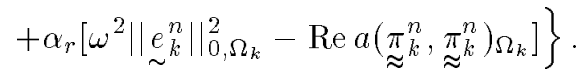


The proof is completed.

We are now ready to show Theorem 3.1 .

Proof of Theorem 3.1. First, we define for $n \geq 1$

$$
\begin{aligned}
& R^{n}=4 \sum_{k=1}^{J}\left\{\alpha_{i}\left[\omega^{-1} \underset{\approx}{A^{-1}} \underset{\approx}{\pi_{k}^{n}} \underset{\sim}{\nu_{k}}, \underset{\approx}{\pi_{k}^{n}} \nu_{\sim}\right\rangle_{\Gamma_{k}}-\operatorname{Im} a\left(\underset{\approx}{\pi}, \underset{\approx_{k}^{n}}{\pi_{\Omega_{k}}}\right]\right. \\
& \left.+\alpha_{r}\left[\omega^{2}\left\|{\underset{\sim}{k}}_{k}^{n}\right\|_{0, \Omega_{k}}^{2}-\operatorname{Re} a\left(\underset{\approx}{\pi},{\underset{\approx}{\pi}}_{k}^{n}\right) \Omega_{k}\right]\right\} .
\end{aligned}
$$

By Lemma 3.4 we get

$$
\begin{aligned}
E^{n+1} & =E^{n}-R^{n} \\
& =E^{0}-\sum_{\ell=1}^{n} R^{\ell} .
\end{aligned}
$$

Next, we want to show that $R^{\ell}$ has a positive lower bound for $\ell \geq 1$, which then implies that $R^{\ell} \longrightarrow 0$ as $\ell \longrightarrow \infty$.

Since $\underset{\approx}{A}$ is positive definite, so is $\underset{\approx}{A^{-1}}$. Hence there is a positive constant $c_{0}$ such that

$$
c_{0}\left|\underset{\approx}{\pi_{k}^{n}} \nu_{\sim}\right|^{2} \leq \underset{\approx}{A^{-1}} \underset{\approx}{\pi_{k}^{n}} \nu_{\sim_{k}} \cdot \underset{\approx}{\pi_{k}^{n}} \nu_{\sim} \leq c_{0}^{-1}\left|\underset{\approx}{\pi_{k}^{n}} \nu_{\sim}\right|^{2}
$$

Notice that

$$
\begin{aligned}
& \operatorname{Re} a\left(\underset{\approx}{\pi_{k}^{n}}, \underset{\approx}{\pi_{k}^{n}}\right)_{\Omega_{k}}=\int_{\Omega}\left[\frac{\mu_{r}}{2|\mu|^{2}}\left|\pi_{\approx}^{n}\right|^{2}-\gamma_{r}\left|\operatorname{tr}\left(\underset{\approx}{\pi_{k}^{n}}\right)\right|^{2}\right] d x, \\
& \operatorname{Im} a(\underset{\approx}{\pi}, \underset{\approx}{\pi})_{\Omega_{k}}^{n}=\int_{\Omega}\left[\frac{-\mu_{i}}{2|\mu|^{2}}|\underset{\approx}{\pi}|^{2}-\gamma_{i}\left|\operatorname{tr}\left(\underset{\approx}{\pi_{k}^{n}}\right)\right|^{2}\right] d x,
\end{aligned}
$$

where

$$
\gamma_{r}=\frac{\lambda_{r}|\mu|^{2}+\mu_{r}\left(|\lambda|^{2} \mid+2 \lambda_{i} \mu_{i}\right)}{4|\mu|^{2}|\lambda+\mu|^{2}}, \quad \gamma_{i}=\frac{\lambda_{i}|\mu|^{2}-\mu_{i}\left(|\lambda|^{2}+\lambda_{r} \mu_{r}\right)}{4|\mu|^{2}|\lambda+\mu|^{2}}
$$

Combining (3.11), (3.13) and (3.14) we get

$$
\begin{aligned}
& R^{n} \geq \sum_{k=1}^{J}\left[\alpha_{i} \omega^{-1} c_{0}\left\|\underset{\approx_{k}}{\pi_{\sim}^{n}}\right\|_{k}\left\|_{0, \Gamma_{k}}^{2}+\frac{\alpha_{i} \mu_{i}-\alpha_{r} \mu_{r}}{2|\mu|^{2}}\right\|{\underset{\approx}{k}}_{k}^{n} \|_{0, \Omega_{k}}^{2}\right. \\
& \left.+\left(\alpha_{r} \gamma_{r}+\alpha_{i} \gamma_{i}\right)\left\|\operatorname{tr}\left(\underset{\approx}{\pi_{k}^{n}}\right)\right\|_{0, \Omega_{k}}^{2}+\alpha_{r} \omega^{2}\left\|\underset{\sim}{e_{k}^{n}}\right\|_{0, \Omega_{k}}^{2}\right] .
\end{aligned}
$$

Since $\gamma_{r}>0$ and $\frac{-\mu_{i}\left(|\lambda|^{2}+\lambda_{r} \mu_{r}\right)}{4|\mu|^{2}|\lambda+\mu|^{2}} \geq \frac{-\mu_{i}}{4|\mu|^{2}}$, choose $\alpha_{r}$ and $\alpha_{i}$ such that $\alpha_{i} \mu_{i}>4 \alpha_{r} \mu_{r}$ with $\alpha_{r}>0$ and $\alpha_{i}>0$, it follows from (3.12) and (3.15) that

$$
\begin{aligned}
& \left\|\underset{\sim}{e_{k}^{\ell}}\right\|_{0, \Omega_{k}} \stackrel{\ell \longrightarrow \infty}{\longrightarrow} 0, \quad \forall k \geq 1, \\
& \left\|\pi_{\approx}^{\ell}\right\|_{0, \Omega_{k}} \stackrel{\ell \longrightarrow \infty}{\longrightarrow} 0, \quad \forall k \geq 1, \\
& \left\|\underset{\approx}{\pi_{k}^{\ell}} \nu_{\sim}\right\|_{0, \Gamma_{k}} \stackrel{\ell \longrightarrow \infty}{\longrightarrow} 0, \quad \forall k \geq 1
\end{aligned}
$$


Finally, choose $\underset{\sim}{v}=\operatorname{div} \underset{\sim}{\pi_{k}^{n}}$ in (3.7.ii) and use (3.16.i) we get

$$
\left\|\operatorname{div} \underset{\approx}{\pi_{k}^{n}}\right\|_{0, \Omega_{k}} \leq \omega^{2}\left\|\underset{\sim}{e_{k}^{n}}\right\|_{0, \Omega_{k}} \stackrel{n \rightarrow \infty}{\longrightarrow} 0, \quad \forall k \geq 1
$$

which together with (3.16.i) and (3.16.ii) completes the proof.

Remark. If the material is elastic ( $\lambda_{i}=\mu_{i}=0$ ), the conclusion of Theorem 3.1 still holds for $\alpha_{r}=0$ and $\alpha_{i}>0$. The above proof, however, is no longer valid. One needs to use a more complicated unique continuation argument. See Feng (1992) for a detailed exposition.

§4. The DD method for mixed finite element approximations. To discretize the algorithm (3.5), we are interested in treating the case in which one subdomain equals one finite element of small diameter (though larger subdomains are permissible). That is, $\left\{\Omega_{j}\right\}$ is a partition of $\Omega$ into individual elements (simplices, rectangles, prisms, tetrahedrons).

Due to the difficulty in constructing the finite element space for the symmetric stress tensor space $\underset{\approx}{H}$, the construction of effective and stable mixed finite element spaces for elasticity problems has proven to be very difficult and has not yet been accomplished in a completely satisfactory manner, especially, for three-dimensional elasticity problems (see Arnold, Douglas \& Gupta (1984) and Brezzi \& Fortin (1991) for a discussion on this point). In this section we will focus on presentating the application of the domain decomposition algorithm by considering the problem in two spatial dimensions.

§4.1. The discrete DD algorithm. Let $\underset{\approx}{H^{h}} \times \underset{\sim}{V^{h}}$ denote a mixed finite element subspace of $\underset{\sim}{H} \times \underset{\sim}{V}$. Several choices of $\underset{\sim}{H^{h}} \times{\underset{\sim}{V}}^{h}$ are acceptable (Brezzi \& Fortin (1991)). Here we only consider the subspace of Johnson \& Mercier (1978) and the family of subspaces of Arnold, Douglas \& Gupta (1984), both of which were constructed by using the composite elements. The global mixed finite element approximation to (2.8) is defined by restricting (2.8) to the finite dimensional subspace $\underset{\approx}{H^{h}} \times \underset{\sim}{V^{h}}$. The mixed finite element subspaces $\underset{\approx}{H^{h}} \times{\underset{\sim}{V}}^{h}$ cited above were originally introduced for stationary elasticity problems which are coercive; it is necessary to show that we can still use these subspaces to approximate the noncoercive problem (2.8). We show this by using the duality argument due to Douglas \& Roberts (1982).

In each space $V^{h}$ in the family of mixed finite element spaces referenced above, the vector functions $\underset{\sim}{v_{h}} \in \underset{\sim}{\sim} \underset{\sim}{\tilde{h}}$ are allowed to be discontinuous across $\Gamma_{j k}$. As a consequence, attempting to impose the transmission condition (3.2) would include a flux conservation error, i.e., (3.1.iv) would not be satisfied unless the approximate solution $\underset{\sim}{v_{h}} \in \underset{\sim}{V^{h}}$ to the discrete analogue of (2.8) is a constant, a uninteresting case. As in Douglas, Paes Leme, Roberts \& Wang (1993), this difficulty can be overcome by hybridization, i.e., by introducing Lagrange multipliers (Brezzi \& Fortin (1991), Douglas, Paes Leme, Roberts \& Wang (1993)) $\left\{\lambda_{j k}\right\}$ on the interface $\left\{\Gamma_{j k}\right\}$.

Let $\underset{\approx}{H^{h}} \times \underset{\sim}{V^{h}}$ be either the Johnson-Mercier space or Arnold-Douglas-Gupta spaces. Let $P_{k}\left(\Gamma_{j \ell}\right)$ denote the space of polynomials of degree less than or equal to $k$ on $\Gamma_{j \ell}$. Set

$$
\underset{\approx}{H_{j}^{h}}=\left.\underset{\approx}{H^{h}}\right|_{\Omega_{j}}, \quad \underset{\sim}{V_{j}^{h}}=\left.\underset{\sim}{V^{h}}\right|_{\Omega_{j}}, \quad \underset{\sim}{M_{j \ell}^{h}}=\underset{\sim}{P_{k}}\left(\Gamma_{j \ell}\right) \quad \text { for } \Gamma_{j \ell} \neq \emptyset .
$$


The (global) mixed finite element approximation to (2.4) is defined as follows: Seek $\left.\underset{\sim}{\sigma_{h}}, \underset{\sim}{u_{h}}\right) \in$ $\underset{\approx}{H^{h}} \times \underset{\sim}{V^{h}}$ such that

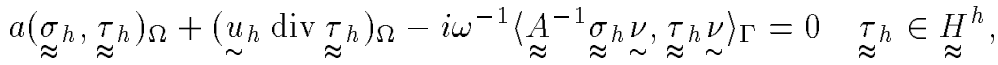

$$
\begin{aligned}
& \left(\operatorname{div} \underset{\sim}{\sigma_{h}},{\underset{\sim}{v} h}_{h}\right)_{\Omega}+\omega^{2}\left({\underset{\sim}{u}}_{h},{\underset{\sim}{v}}_{h}\right)_{\Omega}=-\left(\underset{\sim}{f},{\underset{\sim}{v}}_{h}\right)_{\Omega}, \quad{\underset{\sim}{\sim}}_{h} \in \underset{\sim}{V^{h}} .
\end{aligned}
$$

Theorem 4.1. There exists an $h_{0}>0$ such that, for all $h \in\left(0, h_{0}\right]$, Problem (4.1) has a unique solution $\left(\underset{\sim}{\sigma_{h}}, u_{\sim}\right) \in \underset{\sim}{H}{ }^{h} \times \underset{\sim}{V^{h}}$. Moreover, suppose that $\underset{\sim}{u} \in \underset{\sim}{H^{2}}(\Omega)$, then

$$
\begin{aligned}
& \|\underset{\sim}{\sigma}-\underset{\sim}{\sigma} h\|_{0, \Omega} \leq C\|\underset{\sim}{u}\|_{2, \Omega} h, \\
& \|\underset{\sim}{u}-\underset{\sim}{u}\|_{0, \Omega} \leq C\|\|_{\sim}^{u} \|_{2, \Omega} h^{\min (2, k)},
\end{aligned}
$$

for some positive constant $C$, which depends only on Lamé constants $\lambda_{r}, \lambda_{i}, \mu_{r}, \mu_{i}, \Omega$ and the frequency $\omega$.

A proof of this theorem will be given in the Appendix.

As in the continuous case, it is not difficult to check that the global mixed approximation (4.1) is equivalent to the following hybridized split problem

Seek $\left.\underset{\approx}{\sigma} \sigma_{h j}, u_{h j}, \underset{\sim}{\lambda_{h j k}}\right) \in \underset{\approx}{H_{j}^{h}} \times \underset{\sim}{V_{j}^{h}} \times \underset{\sim}{M_{j k}^{h}}$ such that

$$
\begin{aligned}
& a\left(\underset{\approx}{\sigma_{h j}}, \tau_{h}\right)_{\Omega_{j}}+\left(\underset{\sim}{u_{h j}}, \operatorname{div} \underset{\sim}{\tau_{h}}\right)_{\Omega_{j}}-i \omega^{-1}\left\langle\underset{\approx}{A^{-1}} \underset{\approx}{\sigma_{h j}} \underset{\sim}{\nu_{j}}, \underset{\approx}{\tau_{h}} \nu_{j}\right\rangle_{\Gamma_{j}}
\end{aligned}
$$

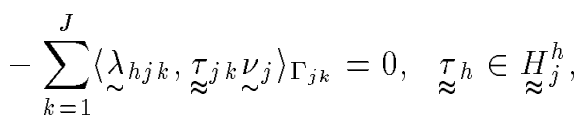

$$
\begin{aligned}
& \left(\operatorname{div} \underset{\sim}{\sigma_{h j}},{\underset{\sim}{v}}_{h}\right)_{\Omega_{j}}+\omega^{2}\left({\underset{\sim}{u}}_{h j},{\underset{\sim}{v}}_{h}\right)_{\Omega_{j}}=-\left(\underset{\sim}{f},{\underset{\sim}{v}}_{h}\right)_{\Omega_{j}},{\underset{\sim}{v}}_{\sigma} \in \underset{\sim}{V_{j}^{h}},
\end{aligned}
$$

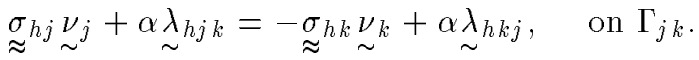

for $j, k=1,2, \ldots, J$. The equivalence is understood as follows: $\left.\underset{\sim}{\sigma}\right|_{\Omega_{j}}=\underset{\sim}{\sigma} h j$ and $\left.\underset{\sim}{u_{h}}\right|_{\Omega_{j}}=u_{\sim} u_{h j}$ where $\left(\underset{\sim}{\sigma_{h}},{\underset{\sim}{u}}_{h}\right)$ is the solution of (4.1).

Again, the constraint (4.3.iii) is equivalent to the discrete consistency conditions analogous to $(3.2)$.

Based on the hybrid formulation (4.3), we define the domain decomposition iterative procedure analogous to (3.5) as follows: For all $j$ and $k$, choose $\underset{\sim}{\sigma_{h j}^{0}} \in \underset{\sim}{H_{j}^{h}}, \underset{\sim}{u_{h j}^{0}} \in \underset{\sim}{V_{j}^{h}}, \underset{\sim}{\lambda_{h j k}^{0}} \in \underset{\sim}{M_{j k}^{h}}$ arbitrarily, then compute $\left\{\underset{\sim}{\sigma_{h j}^{n}}, \underset{\sim}{u_{h j}^{n}}, \underset{\sim}{\lambda_{h j k}^{n}}\right\} \in \underset{\sim}{H} \times \underset{\sim}{h} \times \underset{\sim}{V_{j k}^{h}} \times \underset{\sim}{M_{j}^{h}}$ recursively by solving the following equations

$$
\begin{aligned}
& a\left(\underset{\approx}{\sigma_{h j}^{n}}, \underset{\approx}{\tau}\right)_{\Omega j}+\left(\underset{\sim}{u_{h j}^{n}}, \operatorname{div} \underset{\approx}{\tau}\right)_{\Omega_{j}}-i \omega^{-1}\left\langle\underset{\approx}{A^{-1}} \underset{\approx}{\sigma_{h j}^{n}} \underset{\sim}{\nu_{j}}, \underset{\approx}{\tau}{\underset{\sim}{\nu}}_{\sim}\right\rangle_{\Gamma_{j}} \\
& -\sum_{k=1}^{J}\left\langle\underset{\sim}{\lambda_{h j k}^{n}}, \underset{\approx}{\tau_{h}}{\underset{\sim}{\nu}}_{j}\right\rangle_{\Gamma_{j k}}=0, \quad \underset{\approx}{\tau_{h}} \in \underset{\approx}{H_{j}^{h}}, \\
& \left(\operatorname{div} \underset{\sim}{\sigma_{h j}^{n}}, \underset{\sim}{\nu_{h}}\right)_{\Omega_{j}}+\omega^{2}\left(\underset{\sim}{u_{h j}^{n}}, \underset{\sim}{\nu_{h}}\right)_{\Omega_{j}}=-\left(\underset{\sim}{f}, \underset{\sim}{\nu_{h}}\right)_{\Omega_{j}}, \quad \underset{\sim}{v_{h}} \in \underset{\sim}{V_{j}^{h}}, \\
& \sigma_{\sigma^{\prime} j}^{n} \nu_{\sim}+\alpha \underset{\sim}{\lambda_{h j k}^{n}}=-\underset{\approx}{\sigma^{n k}} \underset{\sim}{\nu_{k}}+\alpha \underset{\sim}{\lambda_{h k j}^{n-1}}, \quad \text { on } \Gamma_{j k} \text {. }
\end{aligned}
$$


It is easy to see that Theorem 4.1 guarantees that the sequence $\left\{\underset{\sim}{\sigma_{h j}^{n}}, \underset{\sim}{u_{h j}^{n}},{\underset{\sim}{h j k}}_{n}^{n}\right\}_{n=1}^{\infty}$ is well defined for $h \leq h_{0}$.

§4.2. Convergence analysis. This subsection is devoted to show the convergence of the discrete DD algorithm introduced in the previous subsection, which is one of the two main results of this section.

Theorem 4.2. Choose $\alpha$ such that $\alpha_{r}>0, \alpha_{i}>0$ and $\alpha_{i} \mu_{i}>4 \alpha_{r} \mu_{r}$, then for $h \leq h_{0}$ the iterates $\left\{\underset{\sim}{\sigma_{h j}^{n}}, u_{\sim}^{n}, \lambda_{h j k}^{n}\right\}$ defined by (4.3) converges to the solution $\left\{\underset{\sim}{\sigma_{h j},}{\underset{\sim}{u}}_{h j}, \lambda_{h j k}\right\}$ of the hybridized mixed finite element procedure (4.3) in the following sense

$$
\begin{aligned}
& \text { (i). }\left.\underset{\approx}{\sigma} \sigma_{h j}^{n} \longrightarrow \underset{\approx}{\sigma} \sigma_{h j} \equiv \underset{\approx}{\sigma_{h}}\right|_{\Omega_{j}} \quad \text { in } \underset{\approx}{H}\left(\operatorname{div}, \Omega_{j}\right), \\
& \text { (ii). }\left.{\underset{\sim}{u}}_{h j}^{n} \longrightarrow \underset{\sim}{u_{h j}} \equiv{\underset{\sim}{\sim}}_{h}\right|_{\Omega_{j}} \quad \text { in } \underset{\sim}{L^{2}}\left(\Omega_{j}\right) \text {, } \\
& \text { (iii). } \underset{\sim}{\lambda_{h j k}^{n}} \text { and } \underset{\sim}{\lambda_{h j k}^{n}} \longrightarrow{\underset{\sim}{\lambda}}_{h j k} \text { in } \underset{\sim}{L^{2}}\left(\Gamma_{j k}\right) \text {. }
\end{aligned}
$$

The proof of Theorem 4.2 is essentially similar to the proof of Theorem 3.1. Because of this we will only give a sketch of the proof.

Define the error functions

$$
\begin{aligned}
& \underset{\underset{\sim}{\pi}}{n}{ }^{n}=\underset{\sim}{\sigma_{h j}}-\underset{\approx}{\sigma_{h j}^{n}}, \quad \stackrel{\sim}{\sim}_{h j}^{n}={\underset{\sim}{\sim}}_{h j}-{\underset{\sim}{u}}_{h j}^{n},
\end{aligned}
$$

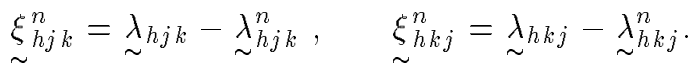

The error equations can be written in the form

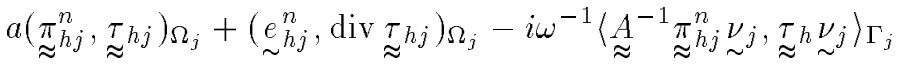

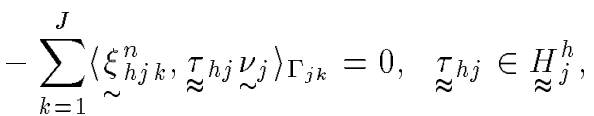

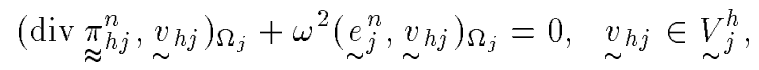

$$
\begin{aligned}
& \underset{\approx}{\pi}{ }^{n}{ }_{\sim}^{\nu_{j}}+\alpha \underset{\sim}{\xi_{h j k}^{n}}=-\underset{\approx}{\pi_{h j k}^{n-1}}+\alpha{\underset{\sim}{h k j}}_{h-1}^{n-1}, \quad \text { on } \Gamma_{j k}
\end{aligned}
$$

Choosing $\underset{\sim}{\tau} \tau_{j}=\underset{\sim}{\pi}{ }_{h j}^{n}$ in (4.5.i) and $\underset{\sim}{v_{h j}}=e_{h j}^{n}$ in (4.5.ii) and adding the resulting equations yields

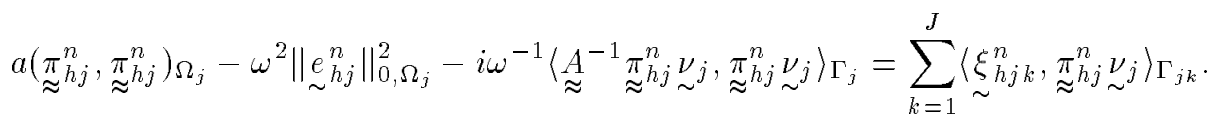

Hence

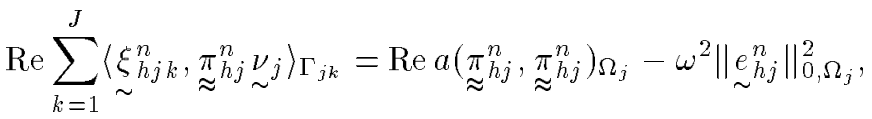

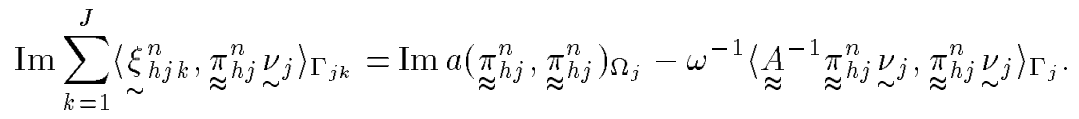


Next, we define

$$
E_{h}^{n}=\sum_{k=1}^{J} \sum_{j=1}^{J} \int_{\Gamma_{k j}}\left|\underset{\sim}{\pi_{h k}^{n}} \underset{\sim}{\nu_{k}}+\alpha \underset{\sim}{\xi_{h k j}^{n}}\right|^{2} d s
$$

it is easy to check that the analogue of Lemma 3.3 holds for $E_{h}^{n}$, that is,

$$
E_{h}^{n+1}=E_{h}^{n}-R_{h}^{n}=E_{h}^{n}-\sum_{\ell=1}^{n} R_{h}^{\ell}
$$

where

$$
\begin{aligned}
& R_{h}^{n}=4 \sum_{k=1}^{J}\left\{\alpha_{i}\left[\omega^{-1} \underset{\approx}{A^{-1}} \underset{\approx}{\pi^{n}}{ }_{\sim}^{\nu_{k}}, \underset{\approx h k}{\pi_{\sim}^{n}} \nu_{k}\right\rangle_{\Gamma_{k}}-\operatorname{Im} a\left(\underset{\approx}{\pi_{h k}^{n}}, \underset{\approx h k}{\pi^{n}}\right)_{\Omega_{k}}\right] \\
& \left.+\alpha_{r}\left[\omega^{2}\left\|e_{\sim}^{n}\right\|_{0, \Omega_{k}}^{2}-\operatorname{Re} a\left(\underset{\approx}{\pi_{h k}^{n}}, \underset{\approx}{\pi_{h k}^{n}}\right)_{\Omega_{k}}\right]\right\} .
\end{aligned}
$$

By an argument similar to one used to establish (3.15), we can show the analogue of (3.15) holds for $R_{h}^{n}$. Hence, choose positive $\alpha_{r}$ and $\alpha_{i}$ such that $\alpha_{i} \mu_{i}>4 \alpha_{r} \mu_{r}$, it follows from (3.15) (for $R_{h}^{n}$ ) that

$$
R_{h}^{n} \geq 4 \sum_{k=1}^{J}\left\{\alpha_{i} \omega^{-1} c_{0}\left\|{\underset{\sim}{\pi} k_{j}}_{\sim}^{\nu_{k}}\right\|_{0, \Gamma_{k}}^{2}+\frac{\alpha_{i} \mu_{i}}{8|\mu|^{2}}\left\|{\underset{\sim}{h k}}_{h k}^{n}\right\|_{0, \Omega_{k}}+\alpha_{r} \omega^{2}\left\|{\underset{\sim}{h k}}_{h k}^{n}\right\|_{0, \Omega_{k}}^{2}\right\}
$$

which together with (4.8.i) implies that

$$
\begin{aligned}
& \left\|\underset{\sim}{e_{h k}^{n}}\right\|_{0, \Omega_{k}} \stackrel{n \longrightarrow \infty}{\longrightarrow} 0, \quad \forall k, \\
& \left\|\pi_{\approx h k}^{n}\right\|_{0, \Omega_{k}} \stackrel{n \longrightarrow \infty}{\longrightarrow} 0, \quad \forall k, \\
& \left\|{\underset{\sim}{h k}}_{h k}^{n} \nu_{\alpha}\right\|_{0, \Gamma_{k}} \stackrel{n \rightarrow \infty}{\longrightarrow} 0, \quad \forall k .
\end{aligned}
$$

Choose $\underset{\sim}{v_{h j}}=\operatorname{div} \underset{\sim}{\pi_{h j}^{n}}$ in (4.5.ii) we get

$$
\left\|\operatorname{div} \underset{\approx}{\pi_{h j}^{n}}\right\|_{0, \Omega_{j}} \leq \omega^{2}\left\|{\underset{\sim}{h j}}_{h j}^{n}\right\|_{0, \Omega_{j}} \stackrel{n \rightarrow \infty}{\longrightarrow} 0
$$

So the convergence for $\underset{\approx}{\sigma_{h j}^{n}}$ and $\underset{\sim}{u_{h j}^{n}}$ is proved.

To show the convergence for $\underset{\sim}{\xi_{h j k}^{n}}$, we will use a similar argument which was used in the proof of Theorem 4.1 in Douglas, Paes Leme, Roberts \& Wang (1993).

Let $\rho_{j}$ denote the ratio of the diameter of $\Omega_{j}$ to the diameter of its inscribed sphere, then for each of the mixed finite element spaces $\underset{\approx}{H^{h}} \times \underset{\sim}{V^{h}}$ we have referenced, it is know that

$$
\left\|\underset{\approx}{\pi_{h j}^{n}}{\underset{\sim}{\nu}}_{j}\right\|_{0, \partial \Omega_{j}} \leq C\left(\rho_{j}, \operatorname{diam} \Omega_{j}\right)\left\|\underset{\approx}{\pi_{h j}^{n}}\right\|_{0, \Omega_{j}}
$$


where $C\left(\rho_{j} \text {, diam } \Omega_{j}\right)^{2} \leq C^{*} h^{-1}$ and $C^{*}$ is independent of $h$ for the quasiuniform triangulations. Thus

$$
\begin{aligned}
& \underset{\approx}{\pi_{h j}}{ }_{\sim}^{\nu_{j}} \longrightarrow 0 \quad \text { strongly in } \underset{\sim}{L^{2}}\left(\partial \Omega_{j}\right), \quad \forall j,
\end{aligned}
$$

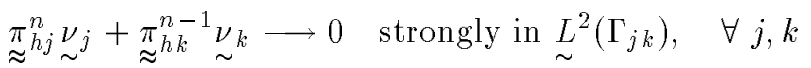

We first consider all boundary elements $\Omega_{j}$ (i.e., $\partial \Omega \cup \partial \Omega_{j} \neq \emptyset$ ). Noticing that for each of the mixed finite element spaces referenced (see Arnold, Douglas \& Gupta (1984)) it is shown that a feasible set of degrees of freedom for $\underset{\approx}{H_{j}^{h}}$ can include

$$
\begin{aligned}
\underset{\sim}{\tau_{h j}}{ }_{\sim}^{\nu_{j}}, & x \in \partial \Omega_{j} \backslash \Gamma_{j 0}, \\
\operatorname{div} \underset{\approx}{\tau_{h j}}, & x \in \Omega_{j} .
\end{aligned}
$$

Moreover, these degrees of freedom can be supplemented, if necessary, in such a way that

$$
\|\underset{\approx}{\tau} h j\|_{0, \Omega_{j}} \leq C\left(\rho_{j}, \operatorname{diam} \Omega_{j}\right)\left(\|\operatorname{div} \underset{\approx}{\tau}\|_{j}\left\|_{0, \Omega_{j}}+\sum_{k=1}^{J}\right\| \underset{\approx}{\tau} h_{j} \underset{\sim}{\nu_{j}} \|_{0, \Gamma_{j k}}\right)
$$

Now, choose $\underset{\approx}{\tau^{n}} \in \underset{\approx j}{H_{j}^{h}}$ on the boundary element $\Omega_{j}$ such that

$$
\begin{aligned}
& \operatorname{div}{\underset{\sim}{\sim} \tau_{h j}^{n}}^{n}=0, \quad \text { in } \Omega_{j}, \\
& \underset{\sim}{\tau_{h j} \nu_{j}}= \begin{cases}\xi_{h j k}^{n}, & \text { on } \Gamma_{j k}, \ell \neq 0, \\
0, & \text { on } \Gamma_{j \ell}, \ell \neq k,\end{cases}
\end{aligned}
$$

then by (4.5.i) and (4.14) we get

$$
\left\|\underset{\sim}{\xi_{h j k}^{n}}\right\|_{0, \Gamma_{j k}}^{2} \leq C\left(\rho_{j}, \operatorname{diam} \Omega_{j}\right)\left\|\underset{\sim}{\pi_{h j}^{n}}\right\|_{0, \Omega_{j}}\left\|\underset{\sim}{\xi_{h j k}}\right\|_{0, \Gamma_{j k}}
$$

Hence

$$
\underset{\sim}{\xi_{h j k}^{n} \longrightarrow 0} \text { strongly in } \underset{\sim}{L^{2}}\left(\Gamma_{j k}\right) \quad \text { if } \Gamma_{j 0} \neq \emptyset,
$$

which and (4.12.ii) imply that

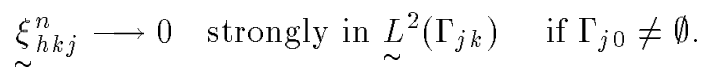

Thus, we have proved the convergence of $\underset{\sim}{\lambda_{h j k}^{n}}$ and $\underset{\sim}{\lambda_{h k j}^{n}}$ on all boundary elements. Next we consider an element having a common face $\Gamma_{j k^{*}}$ with one of the boundary elements. Use div $\underset{\approx}{\tau}$ and $\underset{\sim}{\tau} \nu_{\sim}, j \neq k^{*}$, as degrees of freedom and notice that (4.10.iii) is now replaced by

$$
\underset{\sim}{\xi_{h j k^{*}}^{n} \longrightarrow 0} \text { strongly in } \underset{\sim}{L^{2}}\left(\Gamma_{j k^{*}}\right) \quad \text { as } n \rightarrow \infty,
$$

by repeating the above argument we can show that convergence takes place for $\underset{\sim}{\lambda_{h j k}^{n}}$ and $\underset{\sim}{\lambda_{h k j}^{n}}$ on these elements, as well. The argument can be duplicated until the domain is exhausted. The proof is completed. 
§4.3. Rate of convergence. The objective of this subsection is to derive an upper bound for the rate of convergence of the discrete DD algorithm. Here the rate convergence is measured in terms of the "pseudo energy" norm defined by $E_{h}^{n}$. The main result of this subsection is given by the following theorem.

Theorem 4.3. Under the assumptions of Theorem 4.2, there holds the following estimate

$$
E_{h}^{n+1} \leq(1-C h) E_{h}^{n},
$$

for some positive constant $C$ which is independent of $h$.

Remark. It follows from Theorem 4.3 that the iterative algorithm (4.5) converges at the rate which is less than $(1-C h)$.

Proof of Theorem 4.3. Recall (4.8.i) reads as follows

$$
E_{h}^{n+1}=E_{h}^{n}-R_{h}^{n} .
$$

To prove (4.17), it suffices to show that

$$
E_{h}^{n} \leq(C h)^{-1} R_{h}^{n}
$$

From (4.9) we get

$$
\sum_{k=1}^{J}\left\|{\underset{\approx}{\pi} h}_{h k}\right\|_{0, \Omega_{k}} \leq \frac{2|\mu|^{2}}{\alpha_{i} \mu_{i}} R_{h}^{n} .
$$

So it follows from (4.11) and (4.19) that

$$
\begin{aligned}
\sum_{k=1}^{J}\left\|\underset{\approx}{\pi_{h k}^{n}} \underset{\sim}{\nu_{k}}\right\|_{0, \partial \Omega_{k}}^{2} & \leq \sum_{k=1}^{J}\left[C^{*} h^{-1}\left\|{\underset{\approx}{\pi h}}_{\pi^{n}}^{n}\right\|_{0, \Omega_{k}}^{2}\right] \\
& \leq \frac{2 C^{*}|\mu|^{2} h^{-1}}{\alpha_{i} \mu_{i}} R_{h}^{n} .
\end{aligned}
$$

Finally, using the definition of $E_{h}^{n},(4.16)$ and (4.20) we have

$$
\begin{aligned}
& E_{h}^{n}=\sum_{k=1}^{J} \sum_{j=1}^{J} \int_{\Gamma_{k j}}\left|\underset{\approx}{\pi_{h k}^{n}} \underset{\sim}{\nu_{k}}+\alpha \underset{\sim}{\xi_{h k j}^{n}}\right|^{2} d s \\
& \leq 2 \sum_{k=1}^{J} \sum_{j=1}^{J} \int_{\Gamma_{k j}}\left[\left|{\underset{\sim}{h k}}_{h}^{n} \nu_{\sim}\right|^{2}+|\alpha|^{2}\left|\underset{\sim}{\sum_{h k j}^{n}}\right|^{2}\right] d s \\
& \leq 2 \sum_{k=1}^{J}\left\|{\underset{\sim}{h k}}_{h}^{n} \underset{\sim}{\nu_{k}}\right\|_{0, \partial \Omega_{k}}+2|\alpha|^{2} \sum_{k=1}^{J} \sum_{j=1}^{J}\left\|\underset{\sim}{\xi_{h k j}^{n}}\right\|_{0, \Gamma_{k j}}^{2} \\
& \leq \frac{4 C^{*}|\mu|^{2} h^{-1}}{\alpha_{i} \mu_{i}}\left(1+|\alpha|^{2}\right) R_{h}^{n},
\end{aligned}
$$


which gives (4.18) with $C=\frac{\alpha_{i} \mu_{i}}{4 C^{*}|\mu|^{2}\left(1+|\alpha|^{2}\right)}$. The proof is completed.

Appendix. The purpose of this appendix is to give a proof for Theorem 4.1. We include a proof here since, to the author's knowledge, no proof is available in the literature. Our proof is based on a duality argument introduced in Douglas \& Roberts (1982).

Recall that for each element referenced in Section 4, there exists a projection $\Pi_{h}: \underset{\approx}{{ }^{1}}(\Omega) \rightarrow$ $\underset{\sim}{H^{h}}$ having the properties that

$$
\begin{aligned}
& \left(\operatorname{div}\left(\underset{\sim}{\tau}-\Pi_{h} \underset{\sim}{\tau}\right), \underset{\sim}{v}\right)_{\Omega}=0, \quad \forall \underset{\sim}{v} \in \underset{\sim}{V^{h}}, \\
& \operatorname{div} \underset{\approx}{H^{h}} \subset \underset{\sim}{V^{h}} .
\end{aligned}
$$

Let $P_{h}: \underset{\sim}{V} \rightarrow \underset{\sim}{V^{h}}$ denote the $L^{2}$-projection, a direct consequence of (A.1) is that the projections $\Pi_{h}$ and $\tilde{P_{h}}$ are related by the equation

$$
\operatorname{div} \Pi_{h}=P_{h} \operatorname{div}: \quad \underset{\approx}{H^{1}}(\Omega) \rightarrow \underset{\sim}{V^{h}}
$$

There hold the following approximation properties for $\underset{\sim}{H^{h}} \times \underset{\sim}{V^{h}} \times \underset{\sim}{M^{h}}$ (see Arnold, Douglas \& Gupta (1984))

$$
\begin{array}{ll}
\left\|\underset{\approx}{\tau}-\Pi_{h} \underset{\approx}{\tau}\right\|_{0, \Omega} \leq Q\|\underset{\sim}{\tau}\|_{r, \Omega} h^{r}, & 1 \leq r \leq k+1 \\
\left\|\operatorname{div}\left(\underset{\approx}{\tau}-\Pi_{h} \underset{\approx}{\tau}\right)\right\|_{-s, \Omega} \leq Q\|\operatorname{div} \underset{\approx}{\tau}\|_{r, \Omega} h^{r+s}, & 0 \leq r, s \leq k, \\
\left\|\underset{\sim}{v}-P_{h} \underset{\sim}{v}\right\|_{-s, \Omega} \leq Q\|\underset{\sim}{v}\|_{r, \Omega} h^{r+s}, & 0 \leq r, s \leq k .
\end{array}
$$

Proof of Theorem 4.1. It is easy to see that we only need to derive the error estimate (4.2) since the uniqueness and existence are consequences of the estimate.

Define

$$
\underset{\approx}{\pi}=\underset{\approx}{\sigma}-\underset{\approx}{\sigma} h, \underset{\sim}{e}=u-u_{h}, \underset{\approx}{\theta}=\Pi_{h} \underset{\approx}{\sigma}-\underset{\approx}{\sigma}, \quad \underset{\sim}{q}=P_{h} \underset{\sim}{u}-{\underset{\sim}{\sim}}_{h}
$$

Then, subtracting (4.1) from $(2.8)$ with $\underset{\sim}{g}=0)$ gives the error equations

$$
\begin{aligned}
& \left.a(\underset{\approx}{\pi}, \underset{\approx}{\tau})_{\Omega}+(\underset{\sim}{q}, \operatorname{div} \underset{\approx}{\tau})_{\Omega}-i \omega^{-1} \underset{\approx}{A^{-1}} \underset{\approx \sim}{\pi \nu}, \underset{\approx \sim}{\tau \nu}\right\rangle_{\Gamma}=0, \quad \underset{\approx}{\tau} \in \underset{\approx}{H^{h}}, \\
& (\operatorname{div} \underset{\sim}{\theta}, \underset{\sim}{v})_{\Omega}+\omega^{2}(\underset{\sim}{q}, \underset{\sim}{v})_{\Omega}=0, \quad \underset{\sim}{v} \in{\underset{\sim}{V}}^{h} .
\end{aligned}
$$

Choose $\underset{\sim}{v} \in \underset{\sim}{V^{h}}$ such that $\underset{\sim}{v}=\operatorname{div} \underset{\sim}{\theta}$ in (A.4.ii), we get

$$
\|\operatorname{div} \underset{\approx}{\theta}\|_{0, \Omega} \leq \omega^{2}\left\|_{\sim}^{q}\right\|_{0, \Omega}
$$

Next, take the test function $\underset{\approx}{\tau}=\underset{\approx}{\theta}$ in (A.4.i) to see that

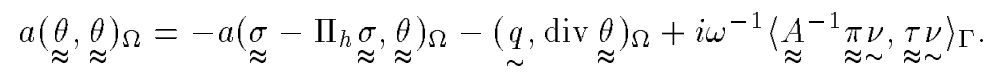


Hence

$$
\operatorname{Re} a(\underset{\approx}{\theta}, \underset{\approx}{\theta})_{\Omega}=\operatorname{Re}\left[-a\left(\underset{\approx}{\sigma}-\Pi_{h} \sigma \underset{\approx}{\sigma}\right)_{\Omega}-(\underset{\sim}{q}, \operatorname{div} \underset{\approx}{\theta})_{\Omega}\right]
$$

consequently, there exists some constant $C_{0}>0$, which depends only on the Lamé constants $\lambda, \mu$ and $\Omega$ such that

$$
\begin{aligned}
& \|\underset{\approx}{\theta}\|_{0, \Omega} \leq C_{0}\left[\omega\|\underset{\sim}{\|}\|_{0, \Omega}+\|\underset{\approx}{\sigma}\|_{1, \Omega} h\right], \\
& \|\underset{\approx}{\pi}\|_{0, \Omega} \leq C_{0}\left[\omega\|\underset{\sim}{q}\|_{0, \Omega}+\|\underset{\approx}{\sigma}\|_{1, \Omega} h\right] .
\end{aligned}
$$

To bound $\|q\|_{0, \Omega}$ by $\|\underset{\sim}{\pi}\|_{0, \Omega}$, we consider the following auxiliary problem: Given $\underset{\sim}{\psi} \underset{\sim}{L^{2}}(\Omega)$, seek $\underset{\approx}{\underset{\sim}{\chi}, w)} \underset{\sim}{\underset{\sim}{H}} \times \underset{\sim}{V}$ such that

$$
\begin{aligned}
& a(\underset{\approx}{\tau}, \underset{\approx}{\chi})_{\Omega}+(\operatorname{div} \underset{\approx}{\tau}, \underset{\sim}{w})_{\Omega}-i w^{-1}\left\langle\underset{\approx}{A^{-1}} \underset{\approx \sim}{\sim} \underset{\sim}{\chi \sim} \underset{\sim}{\chi \nu}\right\rangle_{\Gamma}=0, \underset{\approx}{\tau} \in \underset{\approx}{H}, \\
& (\underset{\sim}{v}, \operatorname{div} \underset{\approx}{\chi})+\omega^{2}(\underset{\sim}{v}, \underset{\sim}{w})_{\Omega}=-(\underset{\sim}{v}, \underset{\sim}{\psi})_{\Omega} \cdot \underset{\sim}{v} \in \underset{\sim}{V},
\end{aligned}
$$

The unique solvability of the above problem is guaranteed by Theorem 2.2. Moreover, from

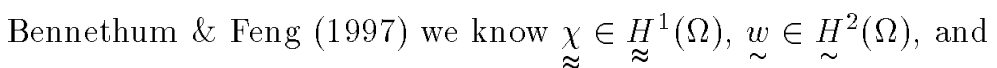

$$
\|\underset{\approx}{\chi}\|_{1, \Omega}+\|\underset{\sim}{w}\|_{2, \Omega} \leq Q\|\psi\|_{0, \Omega}
$$

where $Q$ is some positive constant which depends on $\lambda, \mu, \Omega$ and $\omega$.

It follows from (A.9.ii), (A.1.i) and (A.4.ii) that

$$
\begin{aligned}
(\underset{\sim}{q}, \psi)_{\Omega} & =-(\underset{\sim}{q}, \operatorname{div} \underset{\approx}{\chi})_{\Omega}-\omega^{2}(\underset{\sim}{q}, w)_{\Omega} \\
& =a(\underset{\approx}{\pi}, \underset{\approx}{\chi})_{\Omega}+a\left(\underset{\approx}{\pi}, \prod_{h} \underset{\approx}{\chi}-\underset{\approx}{\chi}\right)_{\Omega}-i \omega^{-1}\left\langle\underset{\approx}{A-1} \underset{\approx \sim}{\operatorname{d}}, \prod_{h} \underset{\approx}{\chi \nu}\right\rangle_{\Gamma}+\left(\operatorname{div} \underset{\approx}{\theta}, P_{h} w\right)_{\Omega} .
\end{aligned}
$$

It follows from (A.9.i)

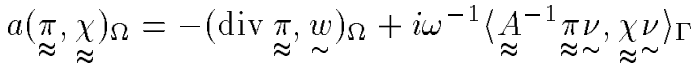

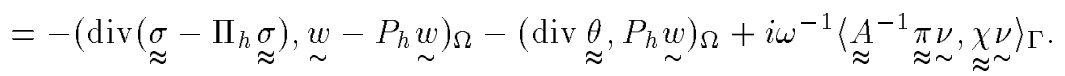

Hence

$$
\left.(\underset{\sim}{q}, \psi)_{\Omega}=a\left(\underset{\approx}{\pi}, \prod_{h} \underset{\approx}{\chi}-\underset{\approx}{\chi}\right)_{\Omega}-\left(\operatorname{div}\left(\underset{\approx}{\sigma}-\prod_{h} \underset{\approx}{\sigma}\right), \underset{\sim}{w_{h}}-P_{h} \underset{\sim}{w}\right)_{\Omega}+i \omega^{-1}\left\langle\underset{\approx}{A-1} \underset{\approx \sim}{\pi \nu}, \underset{\approx}{\chi \chi}-\prod_{h} \underset{\approx}{\alpha}\right) \nu\right\rangle_{\Gamma} .
$$

By the definition of $\Pi_{h}$ (Arnold, Douglas \& Gupta (1984), Douglas \& Roberts (1982)) we have

$$
\int_{e}\left(\underset{\approx}{\sigma}-\Pi_{h} \underset{\approx}{\sigma}\right) \underset{\sim}{\nu} \cdot \underset{\sim}{\varphi} d s=0, \quad \underline{\sim} \in \underset{\sim}{P} P_{k}(e),
$$


for each edge $e$ of every finite element $K$. So

$$
\left.\left\langle\underset{\approx}{A^{-1}} \underset{\approx \sim}{\operatorname{\pi }},\left(\underset{\approx}{\chi}-\Pi_{h} \underset{\approx}{\chi}\right) \underset{\sim}{\nu}\right\rangle_{\Gamma}=\left\langle\underset{\approx}{A}{ }^{-1}\left(\underset{\approx}{\sigma}-\Pi_{h} \underset{\approx}{\sigma}\right) \underset{\sim}{\nu}, \underset{\approx}{\chi}-\Pi_{h} \underset{\approx}{\chi}\right) \underset{\sim}{\nu}\right\rangle_{\Gamma} .
$$

Combining the above two equations we get

$$
\begin{aligned}
\left|(\underset{\sim}{q}, \underset{\sim}{\psi})_{\Omega}\right| & \leq C_{0}\left[\|\underset{\approx}{\|}\|_{0, \Omega}\|\underset{\approx}{\chi}\|_{1, \Omega} h+\|\operatorname{div} \underset{\approx}{\sigma}\|_{0, \Omega}\left\|\left.\right|_{\sim} ^{w}\right\|_{2, \Omega} h^{\min (2, k)}+\|\underset{\approx}{\sigma}\|_{1, \Omega}\|\operatorname{div} \underset{\approx}{\underset{\sim}{\chi}}\|_{0, \Omega} h^{2}\right] \\
& \leq C_{1}\left[\|\underset{\approx}{\pi}\|_{0, \Omega} h+\|\underset{\approx}{\operatorname{div} \sigma}\|_{0, \Omega} h^{\min (2, k)}+\|\underset{\approx}{\sigma}\|_{1, \Omega} h^{2}\right]\|\underset{\sim}{\psi}\|_{0, \Omega},
\end{aligned}
$$

which implies that

$$
\|\underset{\sim}{q}\|_{0, \Omega} \leq C_{1}\left[\|\underset{\approx}{\pi}\|_{0, \Omega} h+\|\operatorname{div} \underset{\approx}{\sigma}\|_{0, \Omega} h^{\min (2, k)}+\|\underset{\approx}{\sigma}\|_{1, \Omega} h^{2}\right] .
$$

Substituting (A.11) into (A.8) yields

$$
\|\underset{\approx}{\pi}\|_{0, \Omega} \leq C_{2}\left[\|\underset{\approx}{\pi}\|_{0, \Omega} \omega h+\|\operatorname{div} \underset{\approx}{\sigma}\|_{0, \Omega} \omega h^{\min (2, k)}++h(1+\omega h)\|\underset{\approx}{\sigma}\|_{1, \Omega}\right] .
$$

Thus, for small $h$ (which depends on $\omega$ ),

$$
\|\underset{\approx}{\pi}\|_{0, \Omega} \leq C_{3}\left[\|\underset{\approx}{\operatorname{div} \sigma}\|_{0, \Omega} \omega h^{\min (2, k)}+h(1+\omega h)\|\underset{\approx}{\sigma}\|_{1, \Omega}\right] .
$$

Finally, the proof is completed by substituting (A.12) into (A.11), which gives the estimate for $\|q\|_{0, \Omega}$.

Acknowledgement. I would like to thank Professor Jim Douglas, Jr. for introducing the problem to me and for his helpful discussions.

\section{REFERENCES}

Arnold, D. N, Douglas, Jr. J., \& C. P. Gupta (1984), A family of higher order finite element methods for plane elasticity, Numer. Math. 45, 1-22.

Bennethum, L .S., \& Feng, X. (1997), A domain decomposition method for solving a Helmholtz-like problem in elasticity based on the Wilson nonconforming element, R.A.I.R.O., Modélisation Math. Anal. Numér. 31, $1-25$.

Bramble, J. H., Pasciak, J. E., Wang, J., \& Xu, J. (1991), Convergence estimates for product iterative methods with applications to domain decomposition, Math. Comp. 57, 1-21.

Brezzi, F., \& Fortin, M. (1991), Mixed and Hybrid Finite Element Methods, Springer-Verlag, New York.

Ciarlet, P. G. (1978), The Finite Element Method for Elliptic Problems, North-Holland, Amsterdam.

Dahlberg, B. E. J., Kenig, C. E., \& Verchota, G. C. (1988), Boundary value problems for the systems of elastostatics in Lipschitz domains, Duke Math. J. 57, 795-818.

Després, B. (1991), Domain decomposition method and Helmholtz problem, in Mathematical and Numerical Aspects of Wave Propagation Phenomena, (G. Cohen, L. Halpern and P. Joly, eds.), SIAM, Philadelphia, $44-52$.

Douglas, Jr., J., Paes Leme, P. J. S., Roberts, J. E., \& Wang, J. (1993), A parallel iterative procedure applicable to the approximate solution of second order partial differential equations by mixed finite element methods, Numer. Math. 65, 95-108.

Douglas, Jr., J., \& Roberts, J. E. (1982), Global estimates for mixed methods for second order elliptic equations, Math. Comp. 1, 91-103. 
Duvaut, G., \& Lions, J. L. (1976), Inequalities in Mechanics and Physics, Springer-Verlag, Berlin.

Feng, X. (1992), On Miscible Displacement in Porous Media and Absorbing Boundary Conditions for Electromagnetic Wave Propagation and on Elastic and Nearly Elastic Waves in the Frequency Domain, Ph. D. Dissertation, Purdue University.

Glowinski, G., \& Wheeler, M. F. (1988), Domain decomposition and mixed finite element methods for elliptic problems, in Domain Decomposition Methods for Partial Differential Equations (R. Glowinski etc., eds.), SIAM, Philadelphia, 144-172.

Hamilton, E. L. (1972), Compressional wave attenuation in marine sediments, Geophysics 37, 620-646.

Johnson, C, \& Mercier, B. (1978), Some equilibrium finite element methods for two-dimensional elasticity problems, Numer. Math. 30, 103-116.

Johnston, D. H., Toksöz, M. N., \& Timur, A. (1978), Attenuation of seismic waves in dry and saturated rocks: II mechanics, Geophysics 44, 691-711.

Le Tallec, P., \& Sassi, T. (1995), Domain decomposition with nonmatching grids: augmented Lagrangian approach, Math. Comp. 64, 1367-1396.

Lions, P. L. (1990), On the Schwartz alternating method III, in the Proceedings of Third International Symposium on Domain Decomposition Method for Partial Differential Equations, (T. Chan etc., eds.), SIAM, Philadelphia, 202-223.

Marini, L. D., \& Quarteroni, A. (1989), A relaxation procedure for domain decomposition methods using finite elements, Numer. Math. 55, 575-598.

Ravazzoli, C. L., Douglas, Jr., J., Santos, J. E., \& Sheen, D. (1992), On the solution of the equations of motion for nearly elastic solids in the frequency domain, Technical Report, Center for Applied Mathematics, Purdue University.

White, J. E. (1965), Seismic Waves, Radiation, Transmission and Attenuation, McGraw-Hill.

Widlund, O. B. (1992), Some Schwarz methods for symmetric and nonsymmetric elliptic problems, in Fifth Conference on Domain Decomposition Methods for Partial Differential Equations, (T. F. Chan etc., eds.), SIAM, Philadelphia, PA, 19-36.

Xu, J. (1992), Iterative methods by space decomposition and subspace correction, SIAM Review 34, 581-613. 